\title{
Wear, Friction, and Microhardness of a Thermal Sprayed PET - Poly (Ethylene Terephthalate) Coating
}

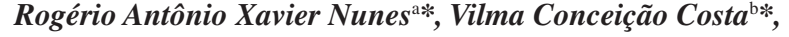 \\ Verônica Maria de Araújo Calado ${ }^{c *}$, José Roberto Tavares Branco ${ }^{\text {a* }}$ \\ ${ }^{\mathrm{a}}$ Fundação Centro Tecnológico de Minas Gerais - CETEC, \\ Rede Temática em Engenharia de Materiais - REDEMAT \\ Universidade Federal de Ouro Preto - UFOP, \\ Universidade do Estado de Minas Gerais - UEMG, \\ ${ }^{\mathrm{b}}$ Fundação Centro Tecnológico de Minas Gerais - CETEC \\ 'Departamento de Química, UFRJ
}

Received: December 4, 2007; Revised: March 13, 2009

\begin{abstract}
The effects of combustion thermal spraying parameters namely combustion pressure, feeding rate, and carrier gas on the wear resistance, friction coefficient, and Knoop hardness of poly (ethylene terephthalate) (PET) films were investigated. The PET coatings were characterized by measuring the wear coefficients by calowear-type testing, the friction coefficients by a pin-on-disk test, and Knoop hardness. The abrasive wear and friction coefficients of the coatings were compared with the values of a post consumer PET bottle chip reference sample. The structural characteristics of the coatings were investigated by X ray diffraction. Statistical analysis of the results allowed for the systematic characterization of the influence of the process variables mentioned above on the coating wear, friction, and microhardness values. Specifically, this study shows that the process parameters affect the wear coefficient and Knoop hardness significantly, but not the friction coefficient. The degree of crystallinity of the PET coatings varied from 20 to $26 \%$.
\end{abstract}

Keywords: thermal spray, PET, tribology

\section{Introduction}

Thermal spray has emerged as one of the most promising coating techniques, producing new materials with tailored chemical, mechanical, and tribological properties. Thermal-sprayed polymer coatings have gained significant attention from many industries, including the petrochemical, automotive, and aircraft industries. They have been used in surface protection against humidity, corrosion, and aggressive chemical products ${ }^{1}$. One of the greatest advantages of thermal spraying compared to other coating techniques is that the coatings can be applied and repaired in the field, and application is not restricted by the size of the surface to be coated ${ }^{1}$. Polymeric materials are expected to be used in corrosion and wear protection with economic advantages over the currently available alternatives ${ }^{2}$.

The thermal spray technique is very versatile due to the wide range of deposition materials and the varying substrate forms and sizes that may be used. In the current state of the art, few polymers have been tested as coatings ${ }^{3}$. Critical process parameters must be selected for each polymer to ensure maximum melting and minimum particle degradation ${ }^{4}$.

Some characteristics of the coating powder, mainly the chemical composition, morphology, molar mass, and particle size, must be adapted to the spraying process parameters and variables. In particular, these include 1) feedstock variables: powder type, size, and shape, carrier gas flow and velocity; 2) torch variables: power, type of thermal energy, gas flow, temperature, cooling; 3 ) jet variables: jet exit velocity and temperature, particle velocity and temperature, particle trajectory; and 4) substrate variables: type and temperature ${ }^{1}$.

Zhang et al. ${ }^{5,6}$ reported that the spraying parameters have significant effects on the heating, melting, and degradation of the particles in the flame, as they affect the molecular structure and behavior of the polymer network and influence the coating properties.

Spray coating properties are determined by the deformation, solidification, and coalescence conditions of the deposited particles, which depend on the substrate properties as well as on the physicalchemical state (temperature, velocity, fusion, and oxidation) of the particles during impact with the substrate. The in-flight behavior of the particles, along with the gas dynamics, may be manipulated by adjusting the process parameters and may directly influence the film microstructure ${ }^{7}$.

Polymeric coatings have excellent tribological properties including low friction coefficients and high wear resistance. The recycling of post-consumer poly(ethylene terephthalate) (PET) in Brazil has significant potential and the use of thermal spraying in tribological applications is promising 8 .

Polymers may be used as dry sliding materials and many studies have been dedicated to examining their friction resistance. Polymers usually exhibit low friction coefficients, with values between 0.1 and $0.5^{9,10}$.

This work intends to contribute to the development of polymer coatings by utilizing the thermal spray of recycled PET for the improvement of the tribological behavior of carbon steel piping. Here, we describe a study of the influences of thermal spraying parameters on the tribological properties of polymeric coatings. Measurement of wear resistance, friction, and Knoop microhardness of these coatings are presented. The results were analyzed using the statistical technique design of experiments (DOE) $)^{11}$. High-wear resistance coatings were produced. 


\section{Materials and Methods}

\subsection{Production of the coatings}

A fine powder of post-consumer PET beverage bottles was obtained by a technique developed at CETEC $^{8}$, from which particle size ranging from 65 to 100 mesh was taken and used as feed stock for a LVOF (Low Velocity Oxy Fuel) thermal spray system. Propane and oxygen were used for combustion while nitrogen gas and compressed air were used as carrier gases.

An ABNT 1020 steel sheet was used as the substrate and the recycled PET powder was used as a feedstock material in the coating process. The substrate was grit-blasted immediately before thermal spraying. Table 1 shows the spraying parameters used during the experiments. A $2^{3}$ complete factorial design of the experiments was used with combustion pressure, feeding rate, and carrier gas as input variables.

\section{Measurements}

\subsection{Structural analysis}

The crystalline structures of the coating, the PET powder, and the PET bottle chip sample were characterized by $\mathrm{X}$ ray diffraction on Shimadzu equipment model XRD-6000 with $\mathrm{CuK}_{\alpha}$ incident radiation over a $2 \theta$ range of $10-50^{\circ}$.

The integral of the total area and of the peak area were also calculated with Origin software to obtain the amorphous fractions and quantify the crystalline phases.

\subsection{Microhardness}

The Knoop microhardness (HK) is given by the ratio of the load to the indentation projected area as:

$$
H K=1.4229 \frac{P}{L^{2}}
$$

where $\mathrm{P}$ is the applied load (kgf) and $\mathrm{L}$ is the larger diagonal indentation $(\mathrm{mm})$.

The Knoop microhardness test was performed on a digital microhardness tester, model FM-700 following ASTM E 384. Knoop microhardness was measured at the cross section of the coatings to minimize the effect of the substrate during indentation. Eight Knoop indentations were performed for each sample with a load of $50 \mathrm{~g}$.

\subsection{Friction coefficient}

The friction coefficient tests were conducted on a pin-on-disk friction tester. The samples were previously ground $(800$ \#) and polished with diamond paste to a mean roughness of $0.5 \mu \mathrm{m}$. The test

Table 1. Thermal spraying parameters.

\begin{tabular}{lc}
\hline \multicolumn{1}{c}{ Parameters } & Values \\
\hline Spraying distance $(\mathrm{mm})$ & 30 \\
Flow rate $(\mathrm{FMR})$ & 30 \\
Preheating temperature $\left({ }^{\circ} \mathrm{C}\right)$ & 100 \\
Carrier gas flow rate $(\mathrm{FMR})$ & 30 \\
Carrier gas pressure $(\mathrm{psi})$ & 30 \\
\hline Variable parameters $-\mathrm{DOE}$ & Values \\
\hline Combustion pressure $(\mathrm{psi})$ & 40 and 50 \\
Powder feeding rate $(\mathrm{g} / \mathrm{min})$ & 2 and 4 \\
Carrier gas & Air and nitrogen \\
\hline
\end{tabular}

parameters were $10 \mathrm{rpm}, 14 \mathrm{~mm}$ trail diameter, $10-\mathrm{N}$ load, 50 turns, and a traveled distance of 2,200 $\mathrm{mm}$. Each sample was measured in triplicate. The samples were weighed before and after testing for calculation of mass loss.

\subsection{Wear test}

Abrasive wear was evaluated by calowear testing, which involves the interaction of a rotating steel sphere with the sample surface with alumina as an abrasive. A semi-spherical crater was formed on the contact surface of the sample. The wear rate was calculated from the crater dimension using Equations 2, 3, and 4:

$$
\begin{aligned}
& S=2 \cdot \pi \cdot R \cdot n \\
& V=\frac{\pi \cdot D^{4}}{64 \cdot R} \\
& Q=\frac{V}{S}
\end{aligned}
$$

where $\mathrm{D}$ is the crater diameter, $\mathrm{n}$ the number of turns, $\mathrm{R}$ the sphere radius, $\mathrm{V}$ the lost volume, $\mathrm{S}$ the sliding distance, and $\mathrm{Q}$ the wear rate $^{12}$.

The wear coefficient (K) was calculated with the Archad equation $^{10}$, where $\mathrm{W}$ is the applied load and $\mathrm{H}$ the hardness.

$$
Q=\frac{K W}{H}
$$

The sliding distances of the sphere were 100, 200, 300, 500, and 1000 turns and the applied load was $13.7 \mathrm{~N}$. Each sample was measured in triplicate. The crater diameter was measured by 3D profilometry.

\section{Results and Discussion}

\subsection{Coating structure}

Using an optical microscope, the presence of bubbles or pores at the substrate/coating interface and in the PET coating medium was not observed. However, they were observed on the coating-free surface. The formation of bubbles is explained in the following way. The final coating temperature was around $410^{\circ} \mathrm{C}$, below the degradation temperature of PET $\left(420^{\circ} \mathrm{C}\right)$. Apparently, the temperature reached by the deposited layers was sufficient for their complete coalescence between the substrate and the coating medium. Particle degradation was insignificant. This will be further discussed with the results of the infrared spectroscopy. Due to the heterogeneous size of the PET particles, the smallest ones probably melted, while the larger ones were semi-melted. During the natural cooling, the coating cools first at the air contact interface with the quick solidification of these layers prior to their coalescence. This results in incomplete contact between layers during the coating process and the formation of surface pores.

Figure 1 shows the $\mathrm{X}$ ray diffractograms of the PET bottle chips and the post-consumer PET powder used in the production of the coatings. The diffractogram of the PET bottle chips shows a diffraction pattern with a single wide peak. In general, the diffraction bands of semi-crystalline polymers are broad and made up of the amorphous phase and reflections of the crystalline planes. The diffraction pattern of the PET bottle chips reveals a semi-crystalline structure with a peak characteristic of the reflection corresponding to the plane with a Miller index (100) for $2 \theta=25.1^{\circ}$. The classical route for the 
production of bottles is based on blowing injected preform molds. This process may lead to molecular orientation by deformation, which suggests a narrow distribution of both the amorphous structure and the crystalline phase. There are several reports on the effect of extrusion (stretching) on the crystallinity and structure orientation PET fibers ${ }^{13}$. The $\mathrm{X}$ ray diffraction results reported by Goshel et al. ${ }^{13}$ indicate the perfect alignment of the orientation of semi-crystalline PET crystals in relation to the stretching direction. A narrow distribution of crystalline orientation is obtained for the parameters of orientation for the normal of the planes (010), (110), and (100) used ${ }^{13}$.

The diffractogram of the post-consumer PET powder (Figure 1) displays reflections characteristic of crystallinity relative to planes (010), (010), (110), and (100) for scattering angles $2 \theta=16.0,17.5$, 22.5 , and $25.5^{\circ}$, respectively. The intensity of the curve observed can be separated into the contributions of the individual crystalline planes and of the amorphous phase. Figure 1 shows these contributions supposing that each peak can be represented by a Gaussian curve. One can observe that the relatively weak intensity of each crystalline plane is covered by the more intense scattering of the amorphous phase. The powder reflections observed are characteristic of PET and have also been observed by other authors ${ }^{13,14}$. The preferential orientation and uniform distribution of the structures have been lost during the PET bottle grinding. The diffraction pattern of the PET powder indicates a random distribution of the planes and the appearance of new crystallinity planes.

The diffractograms of the PET coatings are shown in Figure 2. The post-consumer PET powder used as a spraying raw material was used as a reference sample for comparison with the film diffractograms. Sample 6 presented the lowest intensity values due to its thickness. The peak visible at $2 \theta=45^{\circ}$ represents the presence of iron in the substrate composition, steel 1020.

The $\mathrm{X}$ ray diffractograms of the coatings obtained present a very similar pattern, indicating amorphous and crystalline phases. A two-phase model was used in the determination of the degree of crystallinity, that is, the sample is made up of a crystalline and an amorphous phase without semi-crystalline organization ${ }^{14}$.

Table 2 presents the calculated degree of crystallinity of the PET coatings. The degree of crystallinity of the coatings produced by spraying ranged from 20 to $26 \%$.

Figure 3 displays the Pareto graph of the estimate effects of the thermal spraying parameters on the crystallinity index of the coatings. The main effect of the pressure factor and its $2^{\text {nd }}$ (pressure $x$ feed rate) and $3^{\text {rd }}$ (pressure $\mathrm{x}$ feed rate $\mathrm{x}$ gas) order interactions are significant

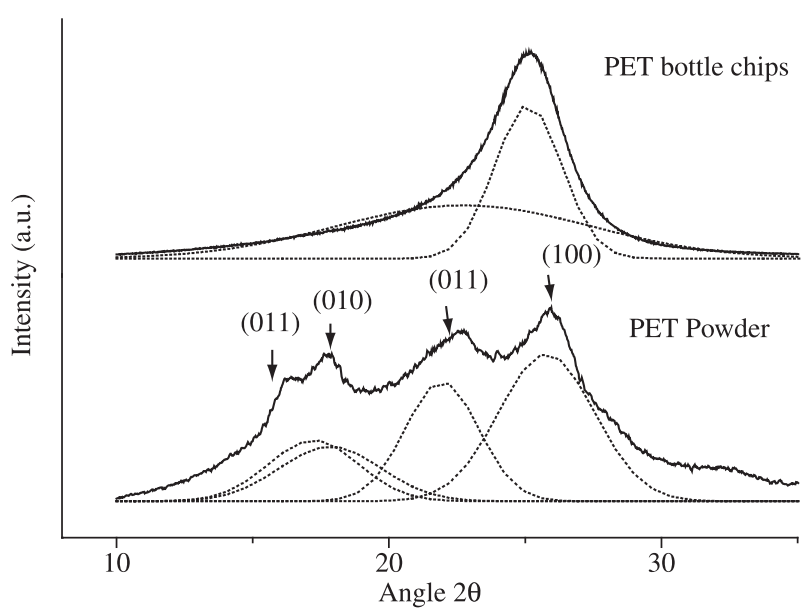

Figure 1. X ray diffractogram of PET bottle chips and feed stock powder. $(p>0.05)$. The velocity of the particles is influenced by the pressure of the fuel gas, and the temperature of the particles depends on the oxygen/fuel ratio. The behavior of the in-flight particles and the gas dynamics was manipulated by adjusting the operation parameters, which directly influences the film microstructure.

\subsection{Microhardness}

The measured microhardness values varied between 18 and $20 \mathrm{HK}$, as can be seen in Table 2. Varacalle et al. ${ }^{15}$ reported the Knoop hardness of polymer coatings obtained by thermal spraying. For polyester, epoxy, and urethane, they found these values to be 23, 18 , and $14 \mathrm{HK}$, respectively.

Plastic deformation occurs in the beginning of the indentation. With an increase of the load, the strain exceeds the elastic limit and the plastic process starts. With a further load increase, the deformation of the material directly under the indenter becomes completely plastic. Some elastic recovery is observed after the release of the load $^{16}$. Generally, hardness increases with a decrease of the applied load, a phenomenon known as the indentation size effect (ISE) ${ }^{17}$. The origin of ISE is directly related to structural factors of the materials under test, including the elastic recovery at indentation and the friction between the indenter and the material ${ }^{17}$.

The statistical results for Knoop microhardness were influenced by the effect of indentation size (ISE), which resulted in high meas-

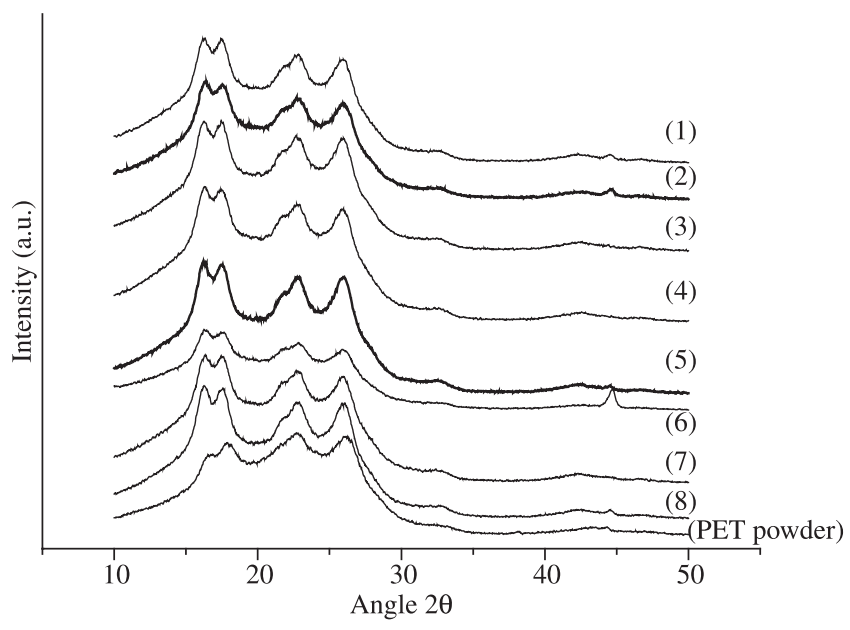

Figure 2. X ray diffractogram of PET coatings and PET powder.

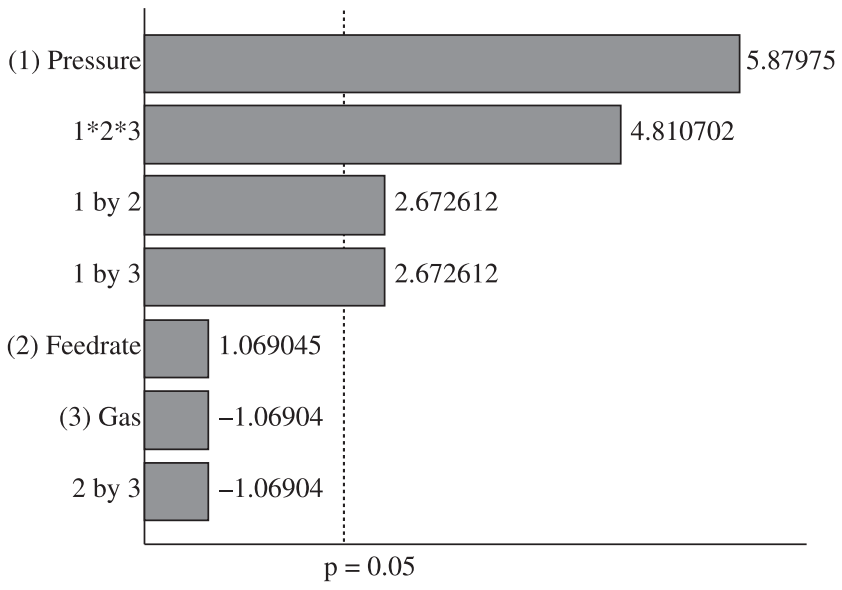

Figure 3. Pareto graph showing the estimated effect of the degree of crystallinity. 
Table 2. Dimensional wear coefficient, friction coefficient, microhardness, and degree of crystallinity.

\begin{tabular}{|c|c|c|c|c|}
\hline Sample & $\begin{array}{l}\text { Dimensional wear coefficient } \\
\left(\mathrm{mm}^{3} / \mathrm{N} . \mathrm{m}\right)\end{array}$ & $\begin{array}{l}\text { Friction coefficient } \\
\qquad(\mu)\end{array}$ & $\begin{array}{l}\text { Microhardness } \\
(\mathrm{HK})\end{array}$ & $\chi c(\%)$ \\
\hline 1 & $2.0 \times 10^{-5}$ & 0.06 & 20.2 & $24 \pm 1$ \\
\hline 2 & $2.8 \times 10^{-5}$ & 0.07 & 19.8 & $21 \pm 1$ \\
\hline 3 & $4.7 \times 10^{-5}$ & 0.06 & 18.9 & $26 \pm 1$ \\
\hline 4 & $4.0 \times 10^{-5}$ & 0.06 & 20.1 & $21 \pm 1$ \\
\hline 5 & $3.0 \times 10^{-5}$ & 0.06 & 19.4 & $25 \pm 1$ \\
\hline 6 & $3.6 \times 10^{-5}$ & 0.06 & 19.8 & $20 \pm 1$ \\
\hline 7 & $5.0 \times 10^{-5}$ & 0.07 & 19.4 & $21 \pm 1$ \\
\hline 8 & $3.8 \times 10^{-5}$ & 0.06 & 19.6 & $23 \pm 1$ \\
\hline PET bottle chips & $1.67 \times 10^{-4}$ & 0.10 & 17.9 & $30 \pm 4$ \\
\hline
\end{tabular}

urement variability. Thus, it was not possible to determine which factors influence microhardness the most.

\subsection{Wear and friction}

Table 2 presents the friction and abrasion wear coefficients of the eight coating samples studied and of the reference sample.

Figure 4 shows the three-D profilometry image of the crater formed on the PET coating in the calowear-type testing with a sliding distance of $2920 \mathrm{~mm}$, equivalent to 500 sliding turns. As shown, a well-defined crater $1.86 \mathrm{~mm}$ in diameter and $35 \mu \mathrm{m}$ in depth was observed. The film was $0.3 \mathrm{~mm}$ thick.

The dimensional wear coefficient values of the coatings produced by spraying are in the order of $10^{-5} \mathrm{~mm}^{3} / \mathrm{Nm}$, in comparison to the wear coefficient value of the control sample, $1.7 \times 10^{-4} \mathrm{~mm}^{3} / \mathrm{Nm}$.

Shipway et al. ${ }^{12}$ reported wear coefficient values $(K)$ for polyethyleneterephthalate glycol (PETG) of $8.5 \times 10^{-3} \mathrm{~mm}^{3} / \mathrm{Nm}$. Friedrich et al. ${ }^{18}$ reported dimensional wear rates $(K)$ for several polymers, including PTFE, PP, and PEEK to be on the order of $10^{-6} \mathrm{~mm}^{3} / \mathrm{Nm}$. Palabiyiik and Bahadur ${ }^{19}$ reported wear rate values of $4.33 \times 10^{-6} \mathrm{~mm}^{3} / \mathrm{Nm}$ for HDPE.

The Pareto graph in Figure 5 shows the estimates of each effect of the factors related to the wear coefficient. The $2^{\text {nd }}$ order interaction (pressure $\mathrm{x}$ feeding rate) was significant. Among the main effects, the feeding rate was significant, as it touches the right size of the line of the level of significance $(\mathrm{p}=0.05)$.

The results obtained suggest that the feeding rate affects the inflight behavior of the particles which, along with the gas pressure, modifies the gas dynamics. The particle velocity is affected by the gas pressure and the particle temperature depends on the oxygen/fuel feeding rate. The feeding rate affects the particle heating, melting, and degradation significantly. At a constant pressure and increased feeding rate, the amount of the in-flight particles will increase, their velocity will decrease, thus increasing residence time in the flame. This increase in residence time may be sufficient to degrade the particles. Larger amounts of particles will require a higher flame temperature to heat and melt them. Consequently, changes in gas pressure and feeding rate will affect the film microstructure significantly, which may be observed through the film wear.

The observed friction coefficient values ranged between 0.06 and 0.07 (Table 2). The friction coefficient values of the PET coatings are slightly lower than that of the reference sample, 0.1 , but this difference is not significant. Unal and Mimaroglu ${ }^{20}$ reported a friction coefficient of 0.06 for PTFE, while Samyn et al. ${ }^{21}$ found a friction coefficient of 0.06 for composite PET/PTFE.

In agreement with the statistical analyses of the friction coefficient, the design of experiments presented that main effects and the 2 nd order interactions were not significant (Figure 6). Thus, the

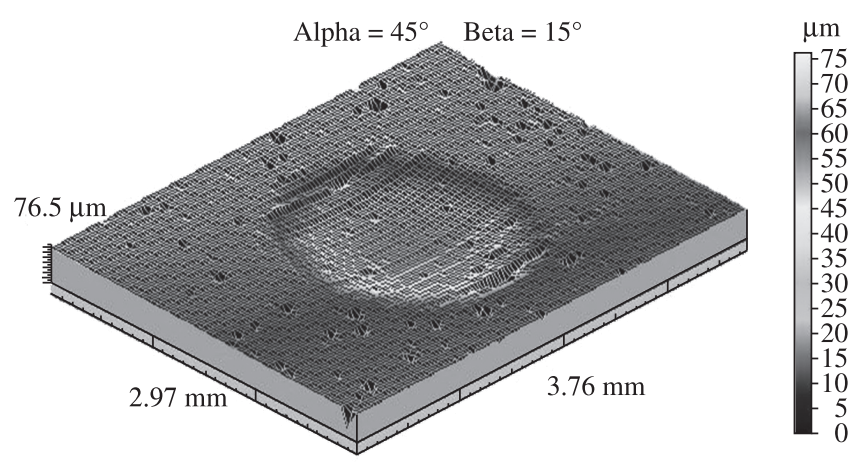

Figure 4. Typical calowear crater formed by calotest, obtained with a profilometer.

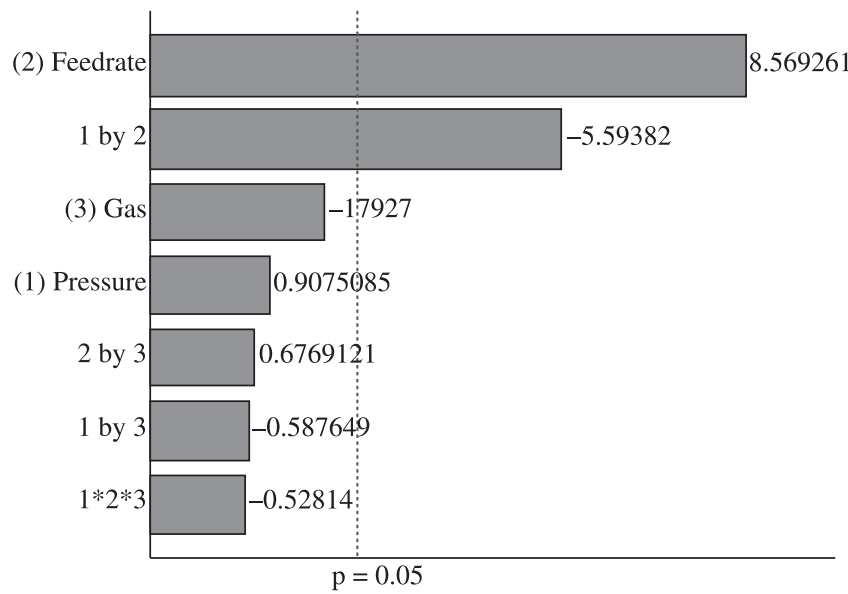

Figure 5. Pareto graph showing the estimated effect of the wear coefficient.

process parameters did not affect the friction coefficient significantly. These results suggest that the viscoelastic effect of the material and the mass transfer influenced the friction coefficient results.

In agreement with the pin-on-disk results for the PET coatings, the friction coefficients remained nearly constant at $10 \mathrm{~N}$, which was also observed by Myshkin et al..$^{22}$ for poly(tetrafluoroethylene) (PTFE), poly(methylmethacrylate) (PMMA), poly(vinyl chloride) (PVC), and polyethylene (PE) surfaces with loads between 10 and 100 N. Other authors obtained similar results with the same materials and also with Teflon (PTFE), poly(methylmethacrylate) (PMMA), poly(vinyl chloride) (PVC), polyethylene (PE), and poly(vinylidene chloride) (PVDC) with loads from 2 to $15 \mathrm{~N}^{22}$. 


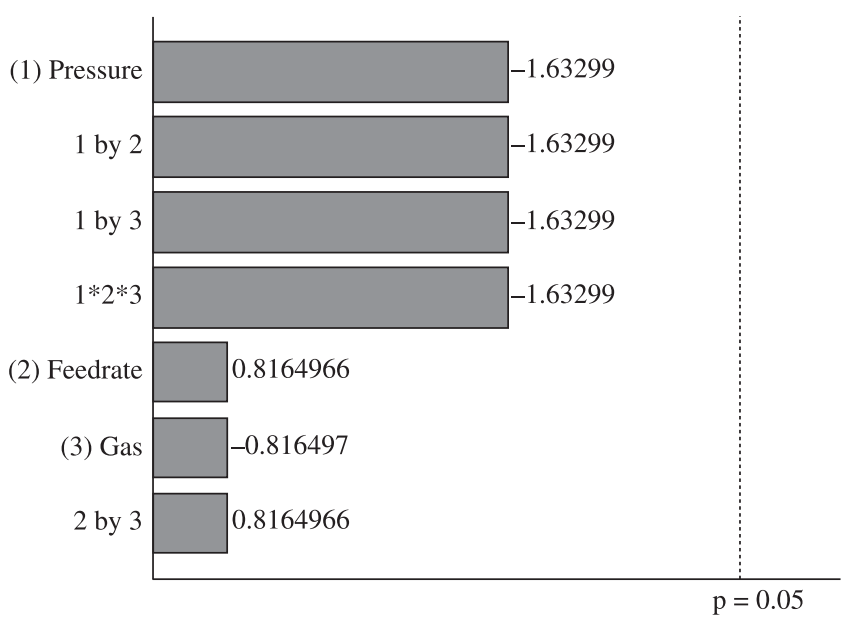

Figure 6. Pareto graph showing the estimated effect of the friction coefficient.

Hutchings ${ }^{10}$ attributes the friction between polymers to two main mechanisms: deformation and adhesion. The cohesive wear mechanism results from the deformation of the material surface by the passage of the counterbody on the polymer surface. The adhesive wear results from shearing between the joints formed in the friction of the bodies. The transfer of material from one surface to another is a distinct characteristic of the contact wear of solid surfaces.

The adhesion responsible for the friction component in polymers results from the break of the hydrogen and van der Walls bonds, which are also responsible for the cohesion of the polymer chains in bulk. Many polymers slide on hard surfaces, mainly on metals, and part of the polymer film is transferred to the surface. The formation and behavior of the transferred films are important factors that affect the friction and wear behaviors of the polymers.

The PET coating surfaces were mechanically deformed by the sphere sliding, which formed a wear track with concentric and continuous lines resulting from the contact between the sphere and the film during the POD assay. The particle fragments found on the sphere surface demonstrate that material transfer occurred.

\section{Conclusions}

The PET coatings obtained in this investigation presented low wear coefficient, with values significantly lower than the dimensional wear coefficient of the control sample $\left(1.7 \times 10^{-4} \mathrm{~mm}^{3} / \mathrm{Nm}\right)$.

The effects of the composition of the coatings, the pressure of the combustion gases, and the powder feeding rate on the coating micro-abrasive wear behavior were significant. The results showed an increase in the wear rate under high pressure and low feeding rate. Low wear coefficients were found at $50 \mathrm{psi}$, with a feeding rate of $2 \mathrm{~g} / \mathrm{min}$, and with compressed air as the carrier gas.

The thermal spraying parameters also influenced the coating microhardness. The indentation size effect on the polymer films affected the microhardness results. It was not possible to identify the cause of these effects.

The low friction coefficients indicate the good tribological properties of the polymer film reported herein.

\section{Acknowledgements}

The authors acknowledge support from CAPES, CETEC and REDEMAT.

\section{References}

1. Petrovicova E, Schadler LS. Thermal spray of polymers. International Materials Reviews. 2002; 47(4):169-190.

2. Davis R. Handbook of thermal spray technology. Materials Park: ASM International; 2004. 53p.

3. Brogan JA. Thermal-spraying of polymer and polymers blends. MRS Bulletin. 2000; 25(7):48-53.

4. Bao Y, Gawne DT, Zhang T. Influence of composition and process parameters on the thermal spray deposition of UHMWPE coatings. Journal of Materials Science. 2005; 40(1):77-85.

5. Zhang T, Gawne DT, Bao Y. The effect of deposition parameters on the degradation of plasma sprayed polymer coatings. In: Berndt CC. (Ed.). Thermal spray: practical solutions for engineering problems. Materials Park: ASM International; 1996. p. 231-237.

6. Zhang G, Liao H, Cherigui M, Davim JP, Coddet C. Effect of crystalline structure on the hardness and interfacial adherence of flame sprayed poly(ether-ether-ketone) coatings. European Polymer Journal. 2007; 43(3):1077-1082.

7. Li M, Shi D, Christofides PD. Diamond jet hybrid HVOF thermal spray: gas-phase and particle behavior modeling and feedback control design. Industrial and Engineering Chemistry Research. 2004; 43(14):3632-3652.

8. Branco JRT, Campos SRV, Duarte LT, Lins VFC. Combustion-thermalsprayed recycled poly(ethylene terephthalate). Journal of Applied polymer Science. 2004; 92(5):3159-3166.

9. Briscoe BJ, Sinha SK. Wear of polymers. Proceedings of the Institution of Mechanical Engineers, Part J: Journal of Engineering Tribology. 2002; 216(6):401-415.

10. Hutchings IM. Tribology: friction and wear of engineering materials. London: Edward Arnold; 1992. 54p.

11. Montgomery DC. Design and analysis of experiments. 5 ed. New York: John Wiley; 2001.

12. Shipway PH, Ngao NK. Microscale abrasive wear of polymeric materials. Wear. 2003; 255(1-6):742-750.

13. Goschel U, Deutscher K, Abetz, V. Wide-angle X ray scattering studies using an area detector: crystalline orientation in semicrystalline PET structures. Polymer. 1996; 37(1):1-6.

14. Xu T, Bin Y, Kurosu H, Matsuo M. Oriented and thermal crystallization of poly(ethylene terephthalate). Colloid and Polymer Science. 2004; 281(7):624-636.

15. Varacalle Jr DJ, Couch KW, Budinger VS. Studies of the flame spraying of polymers. In: Berndt CC. (Ed.). Thermal spray: practical solutions for engineering problems. Materials Park: ASM International; 1996. p. 251-255.

16. Krevelen DWV. Properties of polymers. 3 ed. Amsterdam: Elsevier; 1990

17. Gong J, Wu J, Guan Z. Examination of the indentation size effect in low-load Vickers hardness testing of ceramics. Journal of the European Ceramic Society. 1999; 19(15):2625-2631.

18. Friedrich K, Reinicke R, Zhang Z. Wear of polymer composites. Proceedings of the Institution of Mechanical Engineers, Part J: Journal of Engineering Tribology. 2002; 216(6):415-426.

19. Palabiyik M, Bahadur S. Tribological studies of polyamide 6 and highdensity polyethylene blends filled with PTFE and cooper oxide and reinforced with short glass fibers. Wear. 2002; 253(3-4):369-376.

20. Unal U, Mimaroglu A. Influence of test conditions on the tribological properties of polymers. Industrial Lubrification and Tribology. 2003; 55(4):178-183.

21. Samyn P, Baets P, Schoukens G, Peteghem APV. Large-scale tests on friction and wear of engineering polymers for material selection in highly loaded sliding systems. Materials and Design. 2006; 27(7):535-555.

22. Myshkin NK, Petrokovets MI, Kovalev AV. Tribology of polymers: adhesion, friction, wear, and mass-transfer. Tribology International. 2005; 38(11-12):910-921. 
